\title{
Experimental evaluation on the oil saturation and movability in the organic and inorganic matter of shale
}

\author{
Houjian Gong ${ }^{1,2}$ *, Chaofan Zhu ${ }^{1,2}$, Yinglin Zhang1,2, Zijin Li ${ }^{1,2}$, Qian San ${ }^{1,2}$, \\ Long Xu ${ }^{1,2}$, Yajun $\mathrm{Li}^{1,2}$, Mingzhe Dong ${ }^{3}$, Hassan Hassanzadeh ${ }^{3}$ \\ ${ }^{1}$ Key Laboratory of Unconventional Oil \& Gas Development (China University of \\ Petroleum (East China)), Ministry of Education, Qingdao, Shandong 266580, China \\ ${ }^{2}$ Shandong Key Laboratory of Oilfield Chemistry, School of Petroleum Engineering, \\ China University of Petroleum (East China), Qingdao, Shandong 266580, China \\ ${ }^{3}$ Department of Chemical and Petroleum Engineering, University of Calgary, Calgary, \\ Alberta T2N 1N4, Canada
}
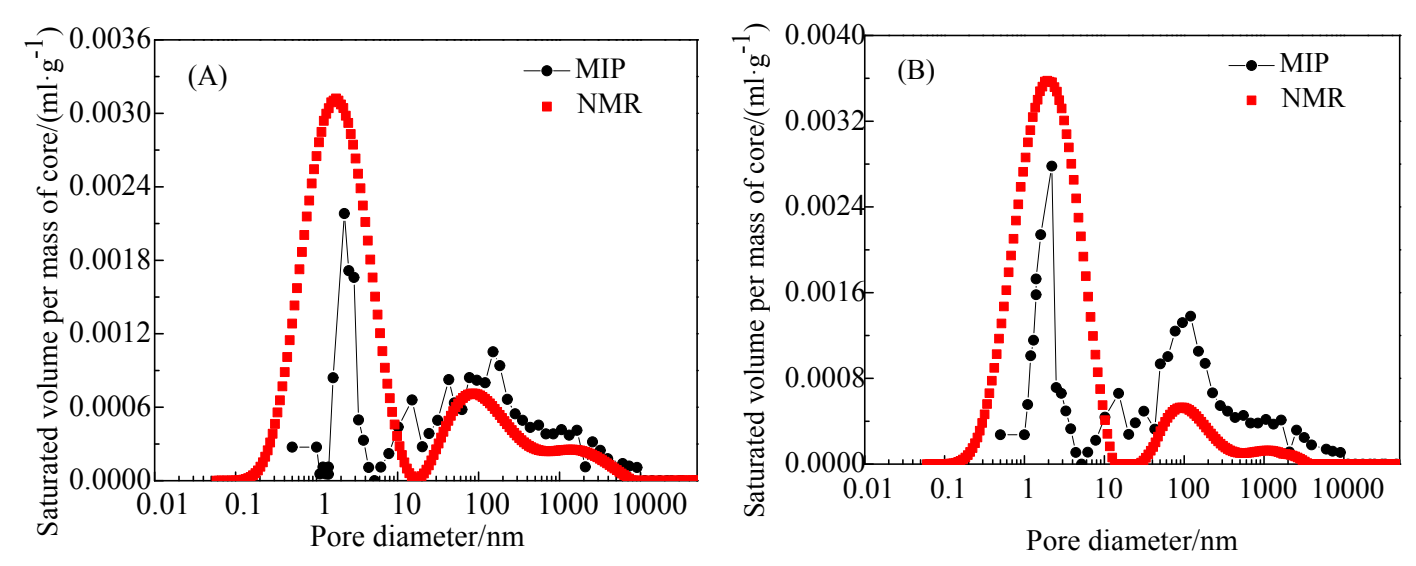

Fig. S1 The comparison of the pore size distribution in shale cores measured by mercury intrusion porosimetry (MIP) and NMR. (A) 9\#; (B) 12\#. 